\title{
Origin of the Asymmetric Magnetization Reversal Behavior in Exchange-Biased Systems: Competing Anisotropies
}

\author{
Julio Camarero, ${ }^{1, *}$ Jordi Sort, ${ }^{2, \dagger}$ Axel Hoffmann, ${ }^{3}$ Jose Miguel García-Martín, ${ }^{4}$ Bernard Dieny, ${ }^{2}$ \\ Rodolfo Miranda, ${ }^{1}$ and Josep Nogués ${ }^{5}$ \\ ${ }^{1}$ Departamento de Física de la Materia Condensada and Instituto "Nicolás Cabrera," Universidad Autónoma de Madrid, \\ 28049 Madrid, Spain \\ ${ }^{2}$ SPINTEC (URA2512 CNRS/CEA), CEA-Grenoble, 38054 Grenoble Cedex 9, France \\ ${ }^{3}$ Materials Science Division and Center for Nanoscale Materials, Argonne National Laboratory, Argonne, Illinois 60439, USA \\ ${ }^{4}$ Instituto de Microelectrónica de Madrid-CSIC, 28760 Tres Cantos, Spain \\ ${ }^{5}$ Institució Catalana de Recerca i Estudis Avançats (ICREA) and Departament de Física, Universitat Autònoma de Barcelona, \\ 08193 Bellaterra, Spain
}

(Received 3 February 2005; published 27 July 2005)

\begin{abstract}
The magnetization reversal in exchange-biased ferromagnetic-antiferromagnetic (FM-AFM) bilayers is investigated. Different reversal pathways on each branch of the hysteresis loop, i.e., asymmetry, are obtained both experimentally and theoretically when the magnetic field is applied at certain angles from the anisotropy direction. The range of angles and the magnitude of this asymmetry are determined by the ratio between the FM anisotropy and the interfacial FM-AFM exchange anisotropy. The occurrence of asymmetry is linked with the appearance of irreversibility, i.e., finite coercivity, as well as with the maximum of exchange bias, increasing for larger anisotropy ratios. Our results indicate that asymmetric hysteresis loops are intrinsic to exchange-biased systems and the competition between anisotropies determines the asymmetric behavior of the magnetization reversal.
\end{abstract}

DOI: 10.1103/PhysRevLett.95.057204

The interfacial exchange coupling between a ferromagnetic (FM) and an antiferromagnetic (AFM) layer has attracted sustained interest over the past decades for both fundamental and technological reasons [1,2]. Experimentally, when a FM-AFM bilayer is field cooled from above the AFM layer's Néel temperature (or grown in the presence of magnetic field), the hysteresis loop is displaced along the field axis by $H_{E}$; i.e., the exchange interaction induces a unidirectional anisotropy in the FM layer. Even though this effect was discovered almost half a century ago [3], there are still ongoing controversies about its underlying basic mechanism [2]. A number of unusual properties are generally observed in exchange-biased FM-AFM systems, among which is an asymmetry in the magnetization reversal [4-18].

The asymmetry of hysteresis loops has been investigated by several experimental techniques that provide information on the vectorial character of the magnetization, such as domain imaging [4-7], polarized neutron reflectometry [8-10], anisotropic magnetoresistance [11,12], vectorial Kerr magnetometry $[13,14]$, or vectorial susceptibility [15]. It has been observed that the magnetization reversal is different at each branch of the (shifted) hysteresis loop and this asymmetry depends on the angle between the external field and the exchange bias direction [5-7]. For example, it has often been reported that while on one side of the loop the reversal takes place by magnetization rotation, on the other side it can take place by domain wall motion or incoherent rotation [8-11]. The origin of the asymmetric reversal has been correlated with the existence of higher order FM anisotropies [7,8,13], disper-
PACS numbers: 75.70.Cn, 75.30.Et, 75.50.Ee, 75.60.Jk

sion of the FM or AFM anisotropy axes [5-7], or irreversibilities due to training [9-12,16,17]. A recent theoretical study has also noted that different angular dependencies of the asymmetry can be obtained for different system parameters, e.g., varying the exchange constants of the AFM layer [18]. However, a general understanding of this effect is still lacking.

In this Letter we study the influence of the anisotropies on the magnetization reversal processes of FM-AFM bilayers with collinear unidirectional, $K_{E}$, and uniaxial, $K_{U}$, anisotropies, by means of high resolution vectorial Kerr magnetometry. A clear asymmetry of the reversal pathways of the two branches of the hysteresis loop is observed for a certain range of measuring angles. The asymmetry depends strongly on $K_{U} / K_{E}$. The onset of asymmetry coincides with that of irreversibility. Numerical simulations based on a modified Stoner-Wohlfarth model confirm that the competition between the unidirectional anisotropy due to the interfacial FM-AFM coupling and the uniaxial anisotropy of the FM layer is the key parameter to understand the experimental observations.

Two series of $\mathrm{Co} / \mathrm{IrMn}$ bilayers, with IrMn thickness $t_{\mathrm{IrMn}}=5 \mathrm{~nm}$ (series I) and $4 \mathrm{~nm}$ (series II) were sputtered at room temperature (RT) on thermally oxidized Si substrates. A buffer layer of $5 \mathrm{~nm}$ Ta was employed to favor [111] texture. The Ta layer was deposited at oblique incidence to promote a uniaxial anisotropy in the FM layer. The thickness of the polycrystalline Co layers, $t_{\mathrm{Co}}$, was varied from 3 to $21 \mathrm{~nm}$. Finally, the samples were capped by $2 \mathrm{~nm}$ of Ta to prevent oxidation. In order to set the unidirectional anisotropy direction, the samples were an- 


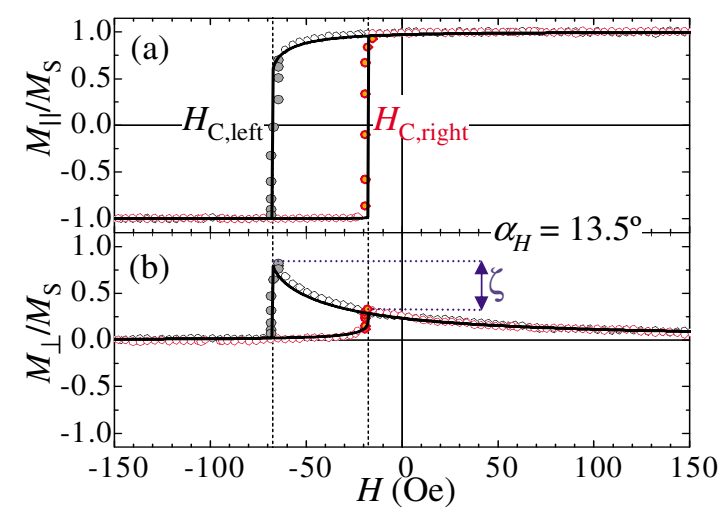

FIG. 1 (color online). Kerr magnetization curves $M(H)$ of a $18 \mathrm{~nm} \mathrm{Co} / 5 \mathrm{~nm}$ IrMn film with the field applied at $13.5^{\circ}$ with respect to the easy axis. This angle was chosen to illustrate the clear asymmetry in the hysteresis loops. The symbols represent experimental $M_{\|}$(a) and $M_{\perp}$ (b) loops, and the continuous lines correspond to simulated curves (see text).

nealed at $420 \mathrm{~K}$ for 30 minutes and field cooled to RT in $H=2.4 \mathrm{kOe}$

with $H$ aligned in the direction of the FM anisotropy. Note that this procedure renders samples with collinear anisotropies, i.e., parallel $K_{U}$ and $K_{E}$. Different interfacial FMAFM coupling values, $J_{\mathrm{F}-\mathrm{AF}}=H_{E} t_{\mathrm{Co}} M_{S}=0.12$ and $0.17 \mathrm{erg} / \mathrm{cm}^{2}$, were found for series I and II, respectively. Since $K_{U}$ is constant and $K_{E}=J / t_{\mathrm{Co}}$, changing $t_{\mathrm{Co}}$ results in different $K_{U} / K_{E}$.

The combination of $p$-polarized light in longitudinal Kerr experiments and the simultaneous detection of the two orthogonal components of the reflected light allows simultaneous determination of the components of the inplane magnetization parallel $\left(M_{\|}\right)$and perpendicular $\left(M_{\perp}\right)$ to the field direction. The difference of the two reflected light components is proportional to $M_{\|}$, whereas the small variations of the sum (i.e., total reflectivity changes) is related to $M_{\perp}$ [19]. Angular dependent hysteresis loops were measured every $1.8^{\circ}$ at RT with $0.5^{\circ}$ angular resolution.

Figure 1 shows representative $M_{\|}(H)$ and $M_{\perp}(H)$ loops for $t_{\mathrm{Co}}=18 \mathrm{~nm}$. Although both decreasing and increasing field branches of the hysteresis loops show smooth reversible (empty symbols in Fig. 1) and sharp irreversible (filled symbols) transitions, a clear asymmetry is observed. $M_{\|}$is more rounded for the decreasing branch than for the increasing branch [Fig. 1(a)]. Interestingly, the asymmetry becomes more obvious for the $M_{\perp}$ loop [Fig. 1(b)]. It is clear that the $M_{\perp}$ peak at $H_{C, \text { left }}$ is significantly larger than the one at $H_{C \text {,right }}$. The fact that $M_{\perp}$ does not reach the saturation magnetization $M_{S}$ means that the magnetization is not stable at $90^{\circ}$. The different peak heights of $M_{\perp}$ for the two branches simply indicate that the angular range for stable magnetization in each branch is different.

Figure 2 summarizes the evolution of the hysteresis loops measured with the magnetic field aligned at different

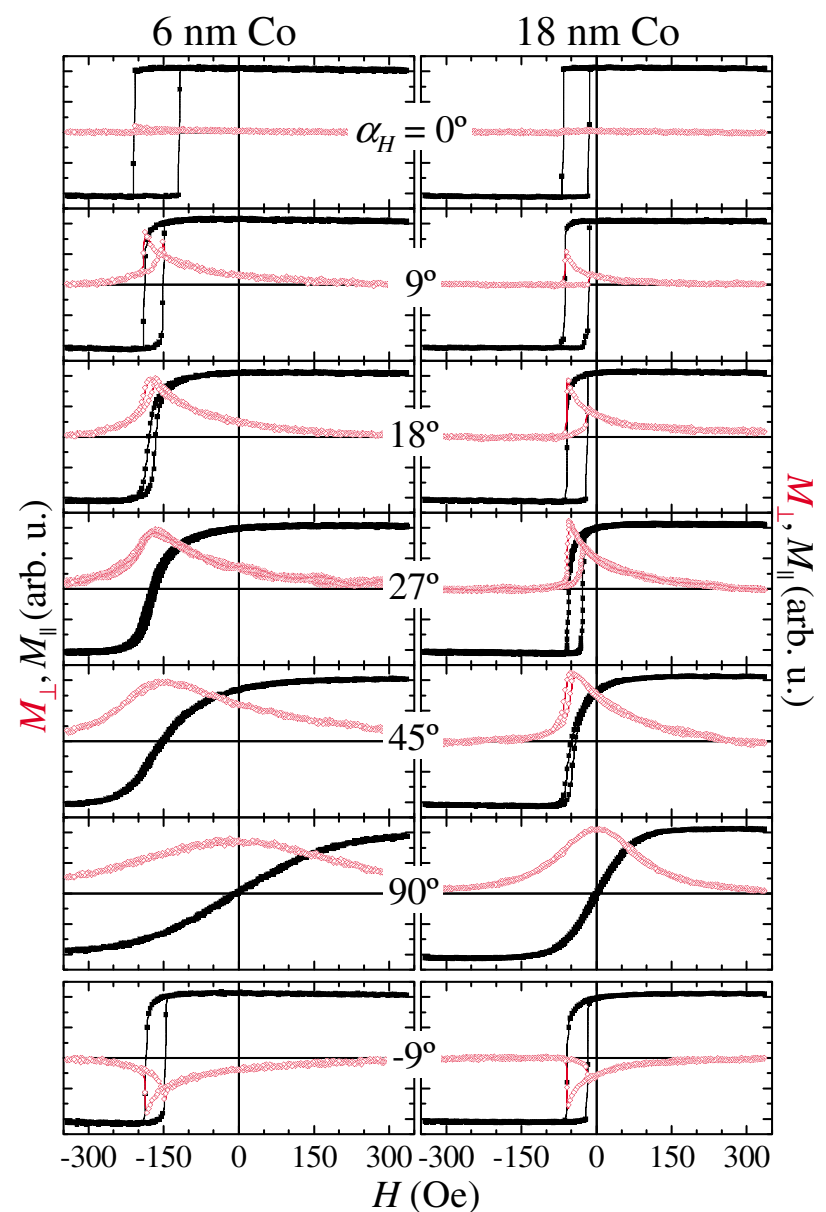

FIG. 2 (color online). Kerr magnetization curves $M(H)$ of $6 \mathrm{~nm} \mathrm{Co} / 5 \mathrm{~nm}$ IrMn (left panels) and $18 \mathrm{~nm} \mathrm{Co} / 5 \mathrm{~nm} \mathrm{IrMn}$ (right panels) films from series I with the field applied at different angles with respect to the easy axis direction. The symbols are experimental loops $M_{\|}$(solid) and $M_{\perp}$ (open).

angles with respect to the easy axis direction $\left(\alpha_{H}=0^{\circ}\right)$ for two selected Co thicknesses from series I, i.e., identical interfacial FM-AFM coupling and different effective FM anisotropy $\left(t_{\mathrm{Co}} K_{U}\right)$. In general, $M_{\perp} \neq 0$ is detected only for $\alpha_{H} \neq 0^{\circ}$, i.e., away from the easy axis direction. In contrast to single FM films, $M_{\perp}$ reverses only in one semicircle (i.e., $M_{\perp}$ remains always positive or negative for the complete hysteresis loop), indicating that this component is sensitive to the exchange field direction (e.g., compare data at $\alpha=9^{\circ}$ and $-9^{\circ}$ in Fig. 2). Either sharp irreversible transitions and/or smoother fully reversible transitions are observed for different angles. There is a range of angles around the easy direction in which the hysteresis loops are found to be irreversible (i.e., $H_{C} \neq$ $0)$ and asymmetric, i.e., $M_{\perp}$ (left) $\neq M_{\perp}$ (right). Note that the asymmetry is much clearer in $M_{\perp}$ and for the thicker FM film, i.e., larger $K_{U} / K_{E}$ ratio. The later might provide the explanation why, in many experimental FM-AFM studies with only $M_{\|}$sensitivity and/or with small FM anisotropy, the asymmetric behavior has not been ob- 
served. Above some critical angle closer to the hard axis direction only smooth reversible transitions are observed, and the hysteresis loops for both components lose the asymmetry. This critical angle increases as the FM thickness increases.

In order to quantify the asymmetry for decreasing and increasing field branches we define $\zeta=\left(M_{\perp \text {, left }}-\right.$ $\left.M_{\perp, \text { right }}\right) / M_{S}$, where $M_{\perp \text {, left }}$ and $M_{\perp \text {,right }}$ are the maximum values of $M_{\perp}$ at $H_{C \text {, left }}$ and $H_{C \text {,right }}$, respectively. From the hysteresis loops in Fig. 2, the angular dependence of the normalized exchange bias, $H_{E}(\alpha)$, coercivity, $H_{C}(\alpha)$, and asymmetry, $\zeta(\alpha)$, can be readily obtained. Figure 3 shows that there is a common range of angles in which $H_{E}(\alpha)$ presents a nonmonotonic behavior typical of collinear unidirectional-uniaxial FM-AFM systems [20], $H_{C}(\alpha)$ exhibits hysteretic (i.e., irreversible) behavior, and the magnetization loops are asymmetric. These features are more pronounced for larger FM thickness. For the other angles (shadowed areas of Fig. 3), a monotonic behavior of the exchange bias, zero coercivity, and zero asymmetry are observed. The critical angle with respect to the easy axes, $\alpha_{C}$, at which the maximum of exchange bias and the onsets of coercivity and asymmetry take place, increases with the FM thickness.

To gain further insight into the asymmetry of the magnetization reversal, we performed numerical simulations based on the Stoner-Wohlfarth model [21] with an addi-

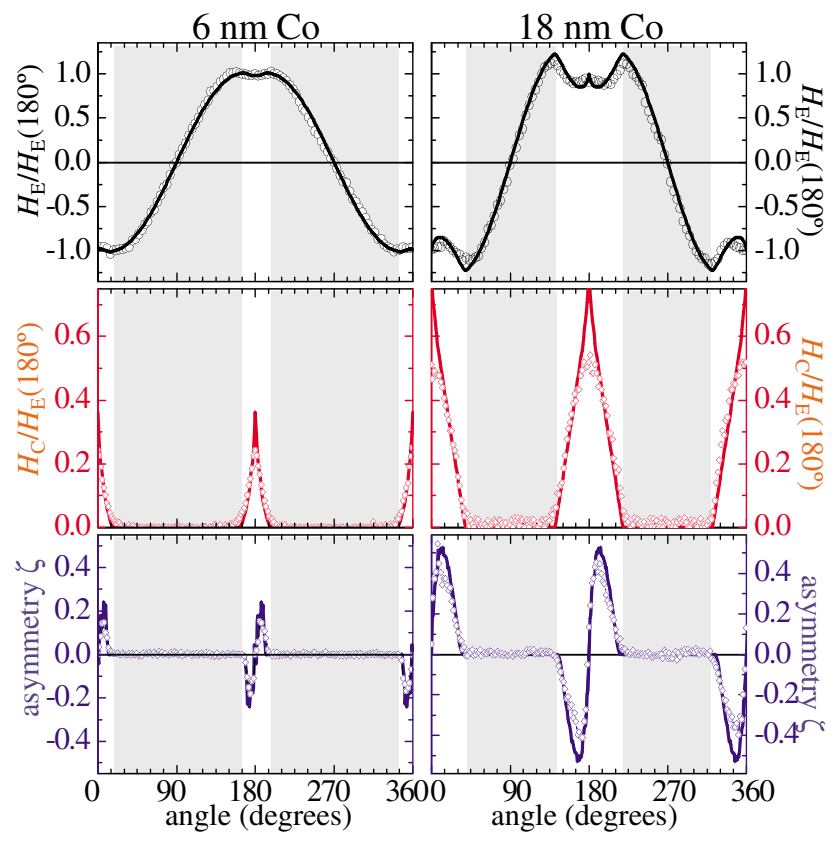

FIG. 3 (color online). Angular dependence of the normalized exchange bias (top panels), coercivity (middle), and asymmetry (bottom) of exchange-coupled $\mathrm{Co} / \mathrm{IrMn}$ films from series I. The lines are simulations of the experimental data (symbols) using a modified Stoner-Wohlfarth model (see text). The range of angles where only reversible processes take place during the reversal are marked by shadowed areas. tional unidirectional $\left(K_{E}\right)$ term, collinear to the uniaxial anisotropy $\left(K_{U}\right)$ [22]. The energy per unit volume of the system is, thus, given by

$$
E=-M_{S} H \cos \theta-K_{E} \cos (\theta-\alpha)-K_{U} \cos ^{2}(\theta-\alpha),
$$

where $M_{S}$ is the saturation magnetization, $H$ is the applied field, and $\theta$ and $\alpha$ are the angles between the applied field and the magnetization and the anisotropy directions, respectively. The parameters $K_{E}$ and $K_{U}$ were determined from the Kerr curves. $K_{E}$ is given by the loop shift at $0^{\circ}$ (i.e., $\left.K_{E}=H_{E} M_{S}\right)$ and $K_{U}\left(K_{U}=\frac{1}{2} H_{K} M_{S}\right.$, where $H_{K}$ is the anisotropy field) can be determined from fitting the hysteresis curve at $90^{\circ}$ [23]. Hysteresis loops are determined numerically via energy minimization of Eq. (1). As shown in Fig. 1, this simple model satisfactorily reproduces the shape of the $M_{\|}$and $M_{\perp}$ hysteresis loops for the different angles.

From the simulated loops, $H_{E}(\alpha), H_{C}(\alpha)$, and $\zeta(\alpha)$ are obtained for different anisotropy ratios, $K_{U} / K_{E}$, corresponding to the different samples. The continuous lines in Fig. 3 show the simulated angular dependence of $H_{E}$, $H_{C}$, and $\zeta$ (with no adjustable parameters), which agrees well with the experimental data, except for the overestimated coercivity around $0^{\circ}$ and $180^{\circ}$. In these regions, irreversible behavior involving domain wall motion and incoherent rotation (e.g., forming domains in directions away from the easy axis [5-7]) might become more important leading to the discrepancy with the calculation. Nevertheless, the extreme values in $H_{E}$ and the onset of nonzero $H_{C}$ and $\zeta$ at $\alpha_{C}$, are nicely reproduced. It is noteworthy that, although this simple approach does not take into account any effects of the AFM, such as microstructure (e.g., grain size or roughness), anisotropy (highlow AFM anisotropy, single crystal-polycrystalline), or AFM domains, the agreement between experimental and simulated results is excellent.

The asymmetry of the reversal can easily be understood if the geometrical asteroid solution of the coherent rotation model is used [24]. A unidirectional anisotropy displaces the asteroid critical curve from the origin [see Fig. 4(a)]. Therefore, if the applied field is not parallel to $K_{E}$, the field sweep line does not pass through the symmetry center of the critical curve. This results in inequivalent switching fields and consequently asymmetric ascending and descending branches of $M_{\perp}(H)$ and $M_{\|}(H)$. Irreversible transitions, i.e., hysteresis, are expected only when the magnetic sweep line passes through the asteroid (filled circles in Fig. 4(a)]. For larger angles, i.e., $\alpha>\alpha_{C}$, the magnetization reversal becomes completely reversible, the field line lies outside the asteroid, and the asymmetry between the two reversals vanishes. The irreversible to reversible transition hence occurs at $\alpha_{C}=\arctan \left(2 K_{U} / K_{E}\right)$. As shown in Fig. 4(b), there is an excellent agreement between the experimental and theoretical $\alpha_{C}$ values.

These results indicate that asymmetric hysteresis loops are intrinsic to exchange bias systems and are not only 


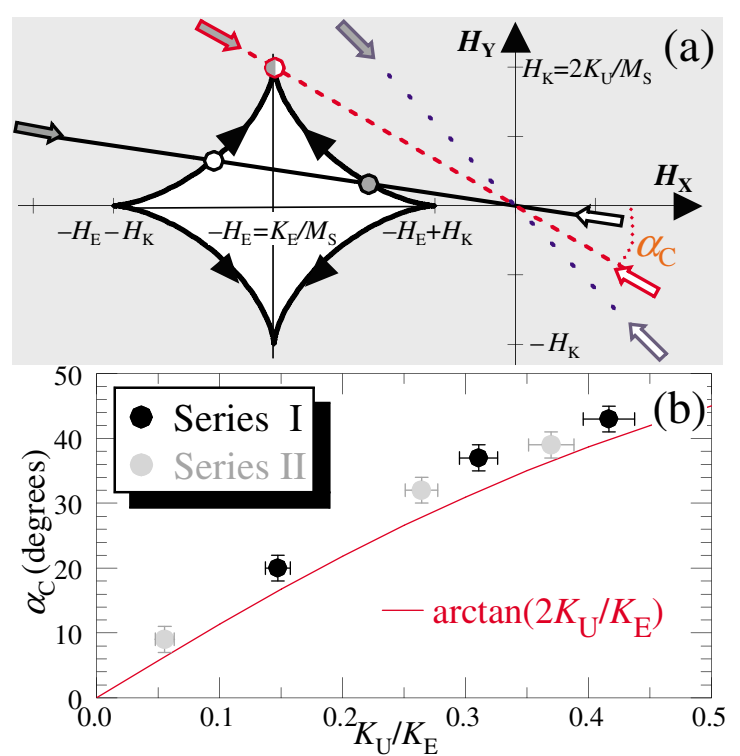

FIG. 4 (color online). (a) Astroid representation of the switching behavior. The continuous, dashed, and dotted straight lines represent fields applied at angles with the easy axis of $10^{\circ}$ (asymmetric-irreversible), $\alpha_{C}$ (critical angle), and $60^{\circ}$ (symmetric-reversible), respectively. The corresponding switching fields of the irreversible transitions in the decreasing (increasing) field branch are depicted by filled white (black) circles in the asteroid. (b) Dependence of the experimental critical angle, $\alpha_{C}$, on the uniaxial/unidirectional anisotropy ratio $K_{U} / K_{E}$ for series I (black circles) and series II (gray circles). The continuous line is the expected evolution from the asteroid representation.

restricted to those FM-AFM systems exhibiting higher order anisotropies. Since perfect alignment is sometimes difficult to obtain experimentally, this may explain the frequent observation of asymmetric hysteresis loops in exchange bias systems with collinear anisotropies. More complex systems, e.g., with higher order (such as cubic) or nonparallel anisotropies or with a dispersion of easy axes, can exhibit types of behavior other than the one described here. Such systems should most likely exhibit asymmetries for a wider range of experimental conditions. In particular, asymmetry could be observed even at $0^{\circ}$. In any case, the asymmetry effects are more pronounced for smaller interface FM-AFM coupling strength and larger FM anisotropies. Consequently, asymmetries might have often been overlooked in systems where one tries to maximize the exchange bias (i.e., using AFM systems with large anisotropy) or minimize the FM anisotropy (i.e., using soft FM materials).

In summary, direct experimental evidence of the relation between the asymmetry of the magnetization reversal in FM-AFM bilayers and the uniaxial and unidirectional anisotropies is shown. The competition between these anisotropies determines the range of angles in which the asymmetry is observed. The onset of both coercivity and asymmetry increases for larger $K_{U}$ or weaker FM-AFM coupling. Hence, by finely tuning the FM anisotropy, the FM-AFM coupling, and the angle of applied magnetic field, it is possible to control with great accuracy both the coercivity and the exchange bias as well as the asymmetry during magnetization reversal of any given FM-AFM bilayer system.

J.C. and J.M.G.-M. acknowledge support through "Ramón y Cajal" contracts from the Spanish MEC. This work was supported in part by the Spanish MEC through Projects No. MAT2003-08627-C02-02, No. MAT200401679, and No. FIS2004-01026, by the U.S. Department of Energy BES-DMS under Contract No. W-31-109-ENG38, and by the European Commission through network NEXBIAS (HPRN-CT 2002-00296).

*Electronic address: julio.camarero@uam.es.

†Present address: Departamento de Física, Universitat Autònoma de Barcelona, 08193 Bellaterra, Spain.

[1] B. Dieny, J. Magn. Magn. Mater. 136, 335 (1994).

[2] J. Nogués and I. K. Schuller, J. Magn. Magn. Mater. 192, 203 (1999).

[3] W.H. Meiklejohn and C.P. Bean, Phys. Rev. 102, 1413 (1956).

[4] V. I. Nikitenko et al., Phys. Rev. B 57, R8111 (1998); V. I. Nikitenko et al., Phys. Rev. Lett. 84, 765 (2000).

[5] A. Kirilyuk et al., J. Appl. Phys. 91, 7745 (2002).

[6] P. Gogol, J. N. Chapman, M.F. Gillies, and F. W. M. Vanhelmont, J. Appl. Phys. 92, 1458 (2002).

[7] J. McCord, R. Schäfer, R. Mattheis, and K.-U. Barholz, J. Appl. Phys. 93, 5491 (2003).

[8] M. R. Fitzsimmons et al., Phys. Rev. Lett. 84, 3986 (2000).

[9] F. Radu et al., J. Magn. Magn. Mater. 240, 251 (2002).

[10] M. Gierlings et al., Phys. Rev. B 65, 092407 (2002); W.-T. Lee et al., Phys. Rev. B 65, 224417 (2002).

[11] C. Leighton et al., Phys. Rev. Lett. 86, 4394 (2001).

[12] E. Pina, C. Prados, and A. Hernando, Phys. Rev. B 69, 052402 (2004).

[13] T. Mewes et al., Phys. Rev. B 65, 224423 (2002).

[14] Z. Y. Liu and S. Adenwalla, Phys. Rev. B 67, 184423 (2003).

[15] L. Spinu et al., Phys. Rev. B 68, 220401(R) (2003).

[16] J. Camarero et al., Phys. Rev. B 67, 020413(R) (2003).

[17] A. Hoffmann, Phys. Rev. Lett. 93, 097203 (2004).

[18] B. Beckmann, U. Nowak, and K. D. Usadel, Phys. Rev. Lett. 91, 187201 (2003).

[19] J. Camarero et al., Phys. Rev. Lett. 91, 027201 (2003).

[20] N. J. Gökemeijer, T. Ambrose, and C. L. Chien, Phys. Rev. Lett. 79, 4270 (1997); H. Xi and R. M. White, J. Appl. Phys. 86, 5169 (1999).

[21] E. C. Stoner and E. P. Wohlfarth, Phil. Trans. R. Soc. A 240, 599 (1948).

[22] A. Hoffmann et al., Phys. Rev. B 67, 220406(R) (2003); S. H. Chung, A. Hoffmann, and M. Grimsditch, Phys. Rev. B 71, 214430 (2005).

[23] M. Grimsditch et al., Phys. Rev. Lett. 90, 257201 (2003).

[24] A. Thiaville, J. Magn. Magn. Mater. 182, 5 (1998). 